\title{
Establishment of the World Health Organization First International Standard for Factor XII, Plasma, Human
}

\author{
Helen V. Wilmot ${ }^{1 *}$, Jason Hockley ${ }^{2}$, Peter Rigsby ${ }^{2}$ and Elaine Gray ${ }^{1}$ \\ ${ }^{1}$ Haemostasis Section, National Institute for Biological Standards and Control, Potters Bar, United Kingdom, \\ ${ }^{2}$ Biostatistics Group, National Institute for Biological Standards and Control, Potters Bar, United Kingdom
}

OPEN ACCESS

Edited by:

Joost Meijers,

University of Amsterdam,

Netherlands

Reviewed by:

Coen Maas,

University Medical Center

Utrecht, Netherlands

Owen McCarty,

Oregon Health \& Science

University, United States

Thomas Renne,

University Medical Center

Hamburg-Eppendorf, Germany

${ }^{*}$ Correspondence:

Helen V. Wilmot

helen.wilmot@nibsc.org

Specialty section:

This article was submitted

to Hematology,

a section of the journal

Frontiers in Medicine

Received: 14 November 2017 Accepted: 07 February 2018

Published: 28 February 2018

Citation:

Wilmot HV, Hockley J, Rigsby P and Gray $E$ (2018) Establishment of the World Health Organization First International Standard for

Factor XII, Plasma, Human. Front. Med. 5:46.

doi: 10.3389/fmed.2018.00046
Until recently, the role of factor XII (FXII) in hemostasis was not considered to be important since patients with FXII deficiency do not present with bleeding. The activation of FXII by agents including mast cells and platelet polyphosphates suggests that it may have a role in thrombogenesis. The inhibition of FXII therefore presents an option for antithrombotic therapy, and antibodies and inhibitors are already in development. Assays for FXII will be required to support these technologies, and an international standard (IS) for FXII would be useful for the development of these methods and for the clinical monitoring of patients. The purpose of this study was to develop an IS for FXII, with values for functional activity (FXII:C) and antigen (FXIl:Ag). Double-spun normal plasma was pooled, filled into siliconized glass ampoules, and freeze-dried to prepare the candidate material. Data from 20 laboratories using the one-stage clotting assay were used to assign the functional activity value in units (u). The antigen value was calculated using data from eight laboratories that carried out antigen assays. Each laboratory was requested to collect two local normal plasma pools. Units of activity and antigen were calculated relative to these pools, as is usual for new coagulation factor analytes. The amount of activity or antigen in $1 \mathrm{ml}$ of normal plasma from each pool was taken to be 1 unit. A total of 566 donors were used across the pools for the FXII:C study and 216 donors for the FXIl:Ag study. The overall geometric mean per ampoule for FXII:C was $0.86 \mathrm{u}$ and for FXIl:Ag was $0.80 \mathrm{u}$. The inter-laboratory variation was 10 and $11 \%$, respectively (expressed as the geometric coefficient of variation). Based on these data, the candidate was deemed suitable for use as an IS for FXII. In 2017, the candidate was established by the World Health Organization (WHO) Expert Committee on Biological Standardization as the WHO first IS for blood coagulation FXII, Plasma (National Institute for Biological Standards and Control code 15/180). The values assigned were 0.86 international units (IU) of functional activity (FXII:C) per ampoule and $0.80 \mathrm{IU} / \mathrm{ampoule}$ of antigen (FXII:Ag).

Keywords: International Standard for factor XII, factor XII functional activity, factor XII antigen, multicenter study, measurement of factor XII

\section{INTRODUCTION}

Factor XII (FXII) is an important coagulation factor in vitro because contact activation of FXII is required for the activated partial thromboplastin time (APTT) assay, a widely used diagnostic test. Historically, the role of FXII in vivo was considered less important, since FXII patients do not suffer from abnormal bleeding and, similarly, FXII-deficient mice are healthy and free from bleeding 
disorders (1). It was later discovered that FXII-deficient mice had defective thrombus formation (2), suggesting that FXII may have a role in thrombosis. Since then, other substances such as platelet polyphosphates (3) and neutrophil extracellular traps (4) have been shown to be in vivo activators of FXII. FXII has also been linked with inflammatory responses such as acute respiratory distress syndrome (5), anaphylaxis, and hereditary angioedema (6). With the knowledge that the depletion of FXII does not affect normal hemostasis, this presents opportunities for therapies targeting FXII to be designed. Various therapies are in development $(7,8)$, and these new technologies require supporting assays for FXII, for which there is currently no International Standard (IS). A plasma IS for FXII functional activity (FXII:C) and antigen (FXII:Ag) would support the development of such assay methods and clinical monitoring of patients.

The processing conditions required to make a standard for FXII are the same as those required for a FXI standard, since both are contact activation factors and exposure to glass or cold results in the activation to FXIIa and FXIa, respectively. The first IS for FXI has just been replaced by the second IS due to stock depletion (9), so this presented an ideal opportunity to use the same processed material to generate the first IS for FXII. This study presents the results of the collaborative study conducted to assign the material with values for functional activity (FXII:C) and antigen (FXII:Ag).

\section{MATERIALS AND METHODS}

\section{Candidate World Health Organization (WHO) First IS for FXII, Plasma [National Institute for Biological Standards and Control (NIBSC) Code 15/180]}

The candidate material $(15 / 180)$ is the same as used for the second IS for FXI, Plasma, details of which have been published elsewhere (9). Briefly, plasma that had been prepared by the centrifugation of whole blood collected into CPD (citrate-phosphate-dextrose) adenine anticoagulant was purchased from the UK Blood Service. The plasma had been subjected to a second centrifugation step to remove cellular material and rapidly frozen at $-70^{\circ} \mathrm{C}$. The individual donations all tested negative for HBsAg, anti-HIV-1 and HIV-2 antibodies, and anti-HCV. Immediately prior to filling, the plasma was thawed at $37^{\circ} \mathrm{C}$ and pooled. A concentration of $40 \mathrm{mM}$ HEPES and $1 \% \mathrm{w} / \mathrm{v}$ glycine was used for the final formulation to improve stability (10). Care was taken to avoid the activation of FXII by cold activation or contact with glass, and the preparation was filled into siliconized glass ampoules and freeze-dried in accordance with recommendations for the preparation of international standards (11). The product had the following physical characteristics: a mean fill mass of $1.0094 \mathrm{~g}$ $(\mathrm{cv}=0.3 \%)$; a mean dry weight of $0.0928 \mathrm{~g}(\mathrm{cv}=0.2 \%)$; a mean residual moisture of $0.605 \%(\mathrm{cv}=13.7 \%)$; and a mean oxygen head space of $0.23 \%(\mathrm{cv}=31.4 \%)$. A total of 6,000 ampoules of $15 / 180$ were produced. The activation status of candidate $15 / 180$ with regard to the presence of FXIIa was assessed. Using the nonactivated partial thromboplastin time (NAPTT) (carried out as described (12) except that FXII-deficient plasma was used), it was found that 15/180 did not shorten NAPTT of FXII-deficient plasma. In addition, results from FXIIa (pre-kallikrein activator) assays (13) indicated undetectable levels of FXIIa. While these test data do not preclude the presence of FXIIa in 15/180, the level present would be unlikely to affect potency estimates of FXII. The long-term stability of $15 / 180$ has been assured by accelerated degradation studies where ampoules of product stored at elevated temperatures are assayed relative to ampoules stored at ultra-low temperature $\left(-150^{\circ} \mathrm{C}\right)$. The predicted loss of FXII:C and FXII:Ag per year when the ampoules are kept at a storage temperature of $-20^{\circ} \mathrm{C}$ was 0.0 and $0.024 \%$, respectively.

\section{Samples in the Study}

The candidate material (NIBSC code 15/180) was provided to the participants as coded duplicate samples, A and B. New coagulation factor standards are assigned relative to normal plasma pools, assuming that $1 \mathrm{ml}$ of plasma contains 1 unit of activity or antigen. Therefore, participants were also asked to collect fresh local normal plasma pools for use both fresh (coded P1) and subsequently frozen (coded P2) in the study, for use as the standard. NIBSC in-house studies on both FXII functional activity and FXII antigen have shown that using fresh plasma, compared to the same pool of plasma after freezing, does not produce significantly different results. Therefore, for the participants unable to collect fresh plasma pools, frozen plasma pools could be used as an alternative. Overall, the pools used to assign the functional activity contained plasma from 566 donors, and the pools used for the antigen assignment comprised 216 donors.

\section{Collaborative Study}

There were 20 laboratories from 10 countries (Austria, Canada, Croatia, Denmark, France, Germany, Netherlands, Spain, United Kingdom, and USA) that took part in the FXII:C assignment. Of these, there were seven clinical laboratories, four therapeutics producers, six diagnostics manufacturers, two regulatory laboratories, and one research laboratory. Eight laboratories took part in the FXII:Ag assignment. These laboratories were from Canada, Denmark, France, Germany, United Kingdom, and USA, comprising one clinical laboratory, one diagnostics manufacturer, four therapeutics producers, and two regulatory laboratories.

Participants were asked to assay coded duplicates A and B against local normal plasma pools and FXII:C and/or FXII:Ag using their usual in-house methods. Laboratories were provided with a protocol for the collection of plasma pools and a separate protocol giving guidance for the randomization of samples and for sufficient replication to enable statistical analysis. Four independent assays were requested for each analyte. FXII is part of the intrinsic pathway and is measured using the APTT assay. APTT reagents contain phospholipid and an activator(s) of the contact pathway (such as ellagic acid). When used with FXII-deficient plasma, the clotting time upon the addition of calcium can be used to calculate the FXII present in a given sample, relative to a standard. All participants in the FXII:C study used one-stage clotting assays with an APTT reagent. Thirteen different APTT reagents were used across different instruments 
and sources of deficient plasma. The sources of APTT reagent (together with the activator and the range of activation times used) and deficient plasmas used are listed in Tables $\mathbf{1}$ and $\mathbf{2}$. Table 3 lists the type of plasma pools used in the functional and antigen assays. For the antigen assays, three different commercial kits or paired antibody sets were used, plus one in-house method (14) (Table 4).

TABLE 1 | Activated partial thromboplastin time (APTT) reagents used by the participants for FXII:C assays.

\begin{tabular}{lcc}
\hline APTT reagent (activator) & $\begin{array}{c}\text { Number of } \\
\text { laboratories }\end{array}$ & $\begin{array}{c}\text { Range of activation } \\
\text { times used (s) }\end{array}$ \\
\hline Actin FS (ellagic acid) & 4 & 180 \\
Actin FSL (ellagic acid) & 3 & 180 \\
APTT-SP (silica) & 2 & 300 \\
Cephascreen (ellagic acid) & 1 & 240 \\
Cephen (silica) & 1 & 240 \\
CK Prest (kaolin) & 3 & 240 \\
Dapttin (silica) & 1 & 240 \\
DG-APTT (ellagic acid) & 1 & 300 \\
Pathromtin SL (silica) & 3 & $120-180$ \\
PTT A (silica) & 1 & 240 \\
Siron LS (ellagic acid) & 1 & 240 \\
SynthAFax (ellagic acid) & 2 & 300 \\
SynthASil (silica) & 5 & $180-300$ \\
\hline
\end{tabular}

TABLE 2 | Sources of deficient plasma used by the participants for FXII:C assays.

\begin{tabular}{lc} 
Deficient plasma & Number of laboratories \\
\hline Affinity Biologicals & 3 \\
Dade & 1 \\
Diagnostic Grifols & 1 \\
Hyphen BioMed & 1 \\
Instrumentation Laboratory & 4 \\
Siemens & 7 \\
Stago & 5 \\
Stago (ImmunoDeficient) & 1 \\
Technoclone & 4 \\
Werfen & 1
\end{tabular}

TABLE 3 | Type of plasma pool used by participants for FXII functional and FXII antigen assays.

\begin{tabular}{lcc}
\hline Plasma pool & $\begin{array}{c}\text { Number of laboratories } \\
\text { in FXII:C study }\end{array}$ & $\begin{array}{c}\text { Number of laboratories } \\
\text { in FXII:Ag study }\end{array}$ \\
\hline Commercial frozen & 6 & 3 \\
Local fresh & 11 & 2 \\
Local frozen & 10 & 1 \\
Lyophilized and frozen & 1 & 2 \\
\hline
\end{tabular}

TABLE 4 | List of antigen kit/paired antibodies used by the participants for FXII:Ag assays.

\begin{tabular}{lc}
\hline Antibody/kit source & Number of laboratories \\
\hline FXII-EIA paired antibodies, Affinity Biologicals & 3 \\
Assay Pro AssayMax & 1 \\
Cedarlane paired antibodies & 3 \\
In-house antibodies & 1
\end{tabular}

Raw data from the participants were returned to NIBSC for parallel line analysis (15) with CombiStats software (CombiStats ${ }^{\mathrm{TM}}$, Version 5.0, Council of Europe). A $\log _{10}$ transformation of the assay response was used for the FXII antigen assays and for the FXII functional assays; data from nine laboratories were transformed, with the rest not requiring transformation. The mean potencies were calculated as unweighted geometric means (GMs), and the variability between assays and laboratories is shown as the geometric coefficients of variation $\left[\mathrm{GCV}=\left(10^{s}-1\right) \times 100 \%\right.$, where $s$ is the SD of the $\log _{10}$ transformed potency estimates]. In order to detect any significant outliers, the $\log _{10}$-transformed laboratory mean estimates were subjected to Grubbs' test (16). Appropriate $t$-tests of $\log _{10}$-transformed laboratory mean estimates were used for comparisons between methods. Where a laboratory performed more than one set of assays (e.g., using a different APTT reagent or instrument), these data were treated independently (designated a, b, etc.).

This study was carried out alongside the study on the second IS for FXI, Plasma (9), and the participants' laboratory number was assigned arbitrarily across the two studies.

TABLE 5 | Factor XII:C, the geometric mean (GM) for each laboratory for samples $A$ and $B$ (coded duplicates), combined and analyzed relative to sample P, each laboratory's local plasma pool.

\begin{tabular}{|c|c|c|}
\hline \multicolumn{3}{|c|}{ Samples AB vs Sample P } \\
\hline Laboratory & GM (u/amp) & GCV (\%) \\
\hline 2 & 0.77 & 2.3 \\
\hline 3 & 0.81 & 15.0 \\
\hline 4 & 0.86 & 10.0 \\
\hline $5 a$ & 0.88 & 11.0 \\
\hline $5 b$ & 0.90 & 12.0 \\
\hline $5 c$ & 0.94 & 3.8 \\
\hline $5 d$ & 0.91 & 12.0 \\
\hline 10 & 0.78 & 4.1 \\
\hline 12 & 0.91 & 2.2 \\
\hline 13 & 0.78 & - \\
\hline 17 & 0.82 & 5.8 \\
\hline 18 & 0.90 & 2.3 \\
\hline 20 & 0.95 & 4.7 \\
\hline 21 & 0.90 & 5.4 \\
\hline 22 & 0.95 & 17.0 \\
\hline 23 & 0.86 & 17.0 \\
\hline 24 & 0.73 & 6.5 \\
\hline $25 a$ & 0.97 & - \\
\hline $25 b$ & 1.07 & 12.0 \\
\hline $26 a$ & 0.72 & 7.9 \\
\hline $26 b$ & 0.99 & 13.0 \\
\hline $27 a$ & 0.81 & 6.1 \\
\hline $27 b$ & 0.79 & 1.0 \\
\hline $27 c$ & 0.79 & 5.5 \\
\hline $27 d$ & 0.79 & 6.0 \\
\hline 28 & 0.81 & 4.5 \\
\hline 29 & 0.82 & 10.0 \\
\hline 30 & 0.86 & 20.0 \\
\hline Overall results & 0.86 & 10.0 \\
\hline for $A B$ vs $P$ & (0.82-0.89) & \\
\hline
\end{tabular}

Intra-laboratory variation is shown as GCV (\%). The overall GM and inter-laboratory GCV for $A B$ VS $P$ is also shown. Figures in brackets indicate the $95 \%$ confidence limits. 


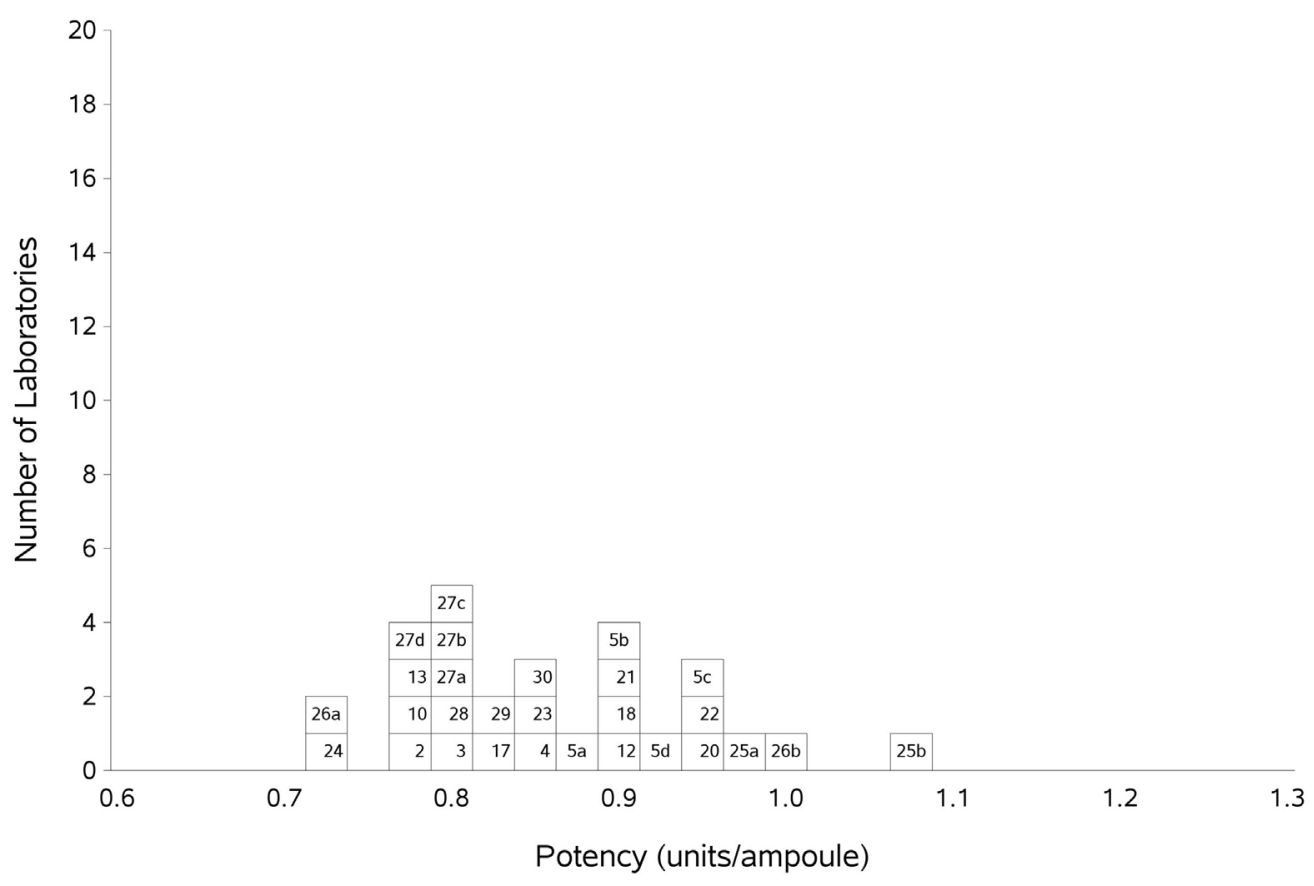

FIGURE 1 | Histogram showing each laboratory's geometric mean (GM) for FXIl:C of samples A and B combined, relative to sample P, the laboratory's local plasma pool. The overall GM was 0.86 IU/ampoule with a GCV of $10.0 \%$.

\section{RESULTS AND DISCUSSION}

\section{FXII Functional Assays}

Participants were requested to prepare two plasma pools (P1 and P2) for use as fresh in two assays and as freeze-thawed pools in a further two assays. Participants who were unable to collect fresh plasma pools were asked to use two different batches of local frozen plasma pools. Results for samples A and $\mathrm{B}$, the coded duplicates, were analyzed relative to $\mathrm{P}$, assuming 1 unit of FXII:C content per $1 \mathrm{ml}$ of plasma pool. The GM for sample A vs $\mathrm{P}$ was 0.86 units per ampoule, with $95 \%$ confidence limits of $0.83-0.89$. The result for sample B was 0.85 units per ampoule, with $95 \%$ confidence limits of $0.82-0.89$. The intra-laboratory variation ranged from 1.7 to $20.0 \%$ for sample A and 1.9 to $19.0 \%$ for sample B, with the majority being less than $10 \%$. Overall, the inter-laboratory GCV was $10 \%$ for each analyte. This variation is to be expected given the different plasma pools used by each laboratory. The results for A and B showed no significant difference, so the results were combined to give overall results for $\mathrm{AB}$ per laboratory (Table 5). The laboratory GM had a close distribution, as demonstrated in Figure 1, and showed that there was good agreement between the laboratories. The overall GM for AB vs $\mathrm{P}$ was $0.86 \mathrm{u}$ /ampoule with an inter-laboratory GCV of $10.0 \%$. Statistical outliers were not detected. Assays performed using fresh plasma pools were compared to those using frozen plasma pools, and there was no significant difference observed $(p=0.859)$. Of the different APTT reagents used, there was no detectable method bias.
TABLE 6 | Factor XII:Ag, the geometric mean (GM) for each laboratory for samples $A$ and $B$ (coded duplicates), combined and analyzed relative to sample P, each laboratory's local plasma pool.

\begin{tabular}{lcr}
\hline Samples AB vs Sample P & & \\
\hline Laboratory & GM (u/amp) & GCV (\%) \\
\hline 6 & 0.84 & 10.0 \\
7 & 0.86 & 7.6 \\
15 & 0.84 & 7.6 \\
17 & 0.70 & 7.6 \\
18 & 0.82 & 9.3 \\
24 & 0.79 & 3.7 \\
$26 a$ & 0.66 & 5.4 \\
$26 b$ & 0.90 & 12.0 \\
28 & 0.84 & 3.9 \\
Overall result & 0.80 & 11.0 \\
for AB vs P & $(0.74-0.87)$ &
\end{tabular}

Intra-laboratory variation is shown as GCV (\%). The overall GM and inter-laboratory GCV for $A B$ vs $P$ is also shown. Figures in brackets indicate the $95 \%$ confidence limits.

\section{FXII Antigen Assays}

As previously, participants were requested to collect two different plasma pools to use as the standard in the assays. Results were analyzed, assuming the FXII:Ag content of $1 \mathrm{ml}$ of plasma to be 1 unit. The overall GM for A against P was $0.80 \mathrm{u} /$ ampoule (95\% confidence limits of $0.74-0.86$ ), compared to 0.81 $\mathrm{u}$ /ampoule (95\% confidence limits of $0.75-0.87$ ) for B against $\mathrm{P}$. The inter-laboratory variation was 10.0 and $11.0 \%$ for A and B, respectively, showing acceptable agreement between laboratories. The results for $\mathrm{A}$ and $\mathrm{B}$, which showed no significant 


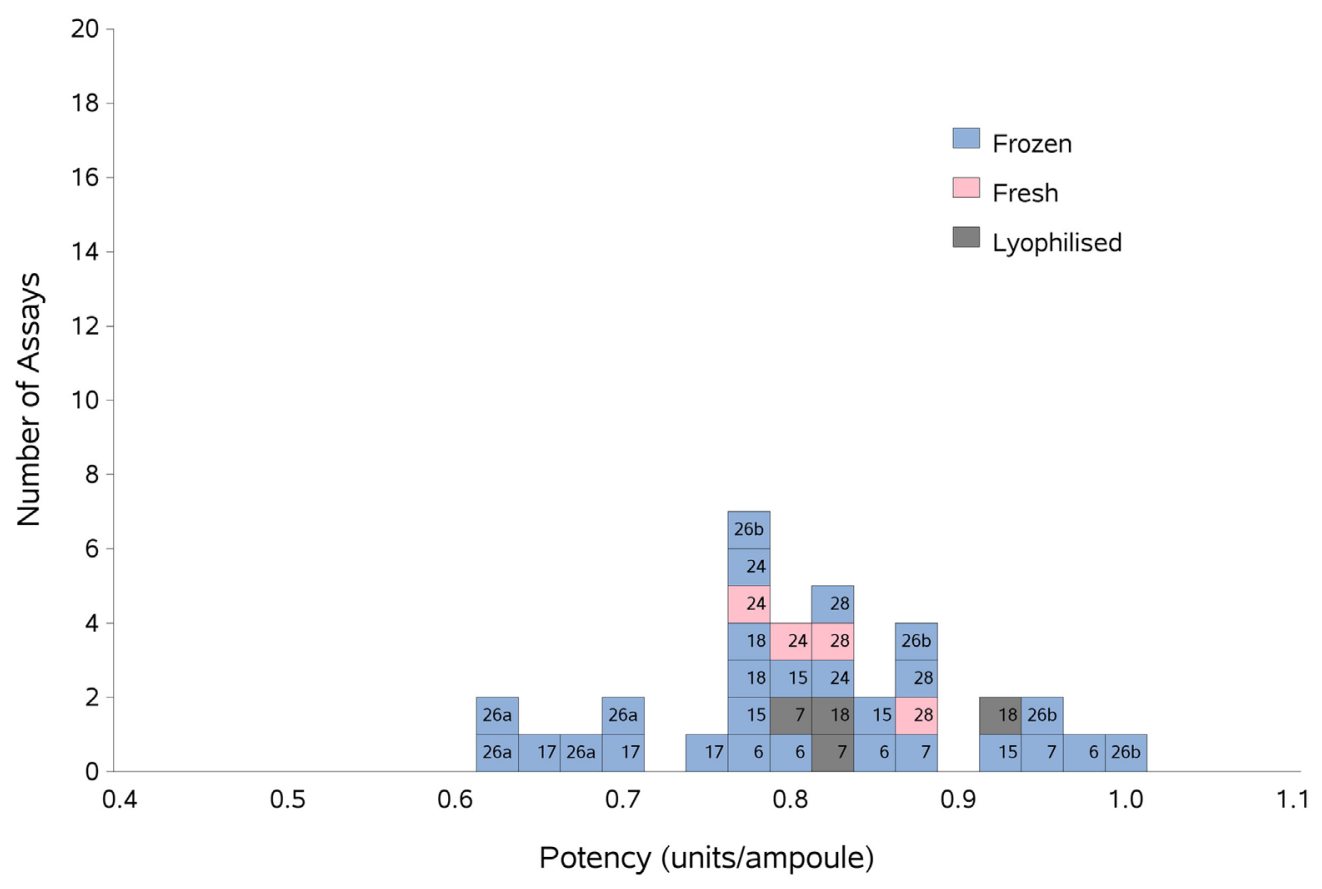

FIGURE 2 | Histogram showing each laboratory's individual assay result for FXII:Ag of samples A and B combined, relative to sample P, the laboratory's local plasma pool. The type of pool used in each assay (fresh, frozen, or lyophilized) is also shown. The overall geometric mean was $0.80 \mathrm{u} / \mathrm{ampoule}$ with a GCV of $11.0 \%$.

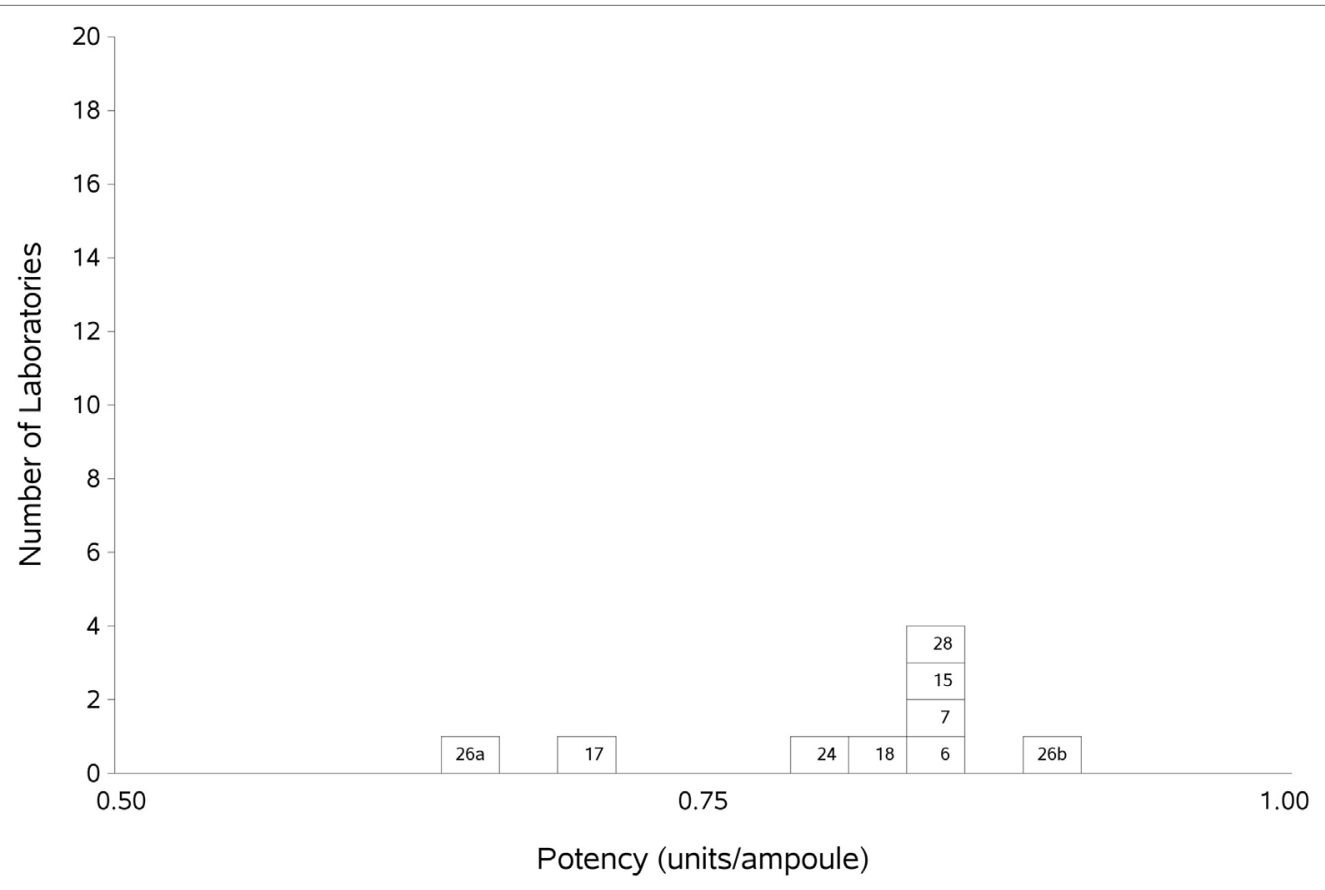

FIGURE 3 | Histogram showing each laboratory's geometric mean for FXII:Ag samples A and B combined, relative to the laboratory's local plasma pool (sample P).

differences, were combined to give a result for $\mathrm{AB}$ overall per laboratory (Table 6). The inter-laboratory variation was $11 \%$, an acceptable value given the inherent variability between plasma pools. No statistical outliers were detected. Figure 2 shows each laboratory's individual assay result, and the laboratory GMs are shown in Figure 3. 
There were not enough laboratories using fresh plasma pools to allow statistical analysis of fresh vs frozen plasma pools; however, Figure 2 shows that the results from the assays performed using fresh plasma pools (assays 1 and 3 from laboratories 24 and 28) fall in the middle of the range of results overall, suggesting that the variability of the values obtained would mostly be due to the source of the plasma pools rather than degradation due to freeze-thawing.

It is important to note that the units for activity and antigen are independent of one another and were value-assigned relative to different plasma pools. The data from the study support the value assignment of 0.86 units per ampoule for FXII:C and 0.80 units per ampoule for FXII:Ag.

\section{CONCLUSION}

The results of this study were presented to the participants and experts nominated by the Scientific and Standardization Committee (SSC) of the International Society on Thrombosis and Haemostasis (ISTH). Both recommended that the candidate 15/180 (the second IS for FXI, Plasma) be used as an IS for FXII:C and FXII:Ag. In October 2017, the WHO ECBS established 15/180 as the first IS for FXII: plasma, human, with assigned values of 0.86 international unit (IU)/amp for FXII functional activity (FXII:C) and $0.80 \mathrm{IU} / \mathrm{amp}$ for FXII antigen (FXII:Ag).

\section{AUTHOR CONTRIBUTIONS}

HW, JH, PR, and EG designed the study. HW and EG organized the study and wrote the manuscript. JH and PR analyzed the data.

\section{REFERENCES}

1. Pauer H, Renné T, Hemmerlein B, Legler T, Fritzlar S, Adham I, et al. Targeted deletion of murine coagulation factor XII gene-a model for contact phase activation in vivo. Thromb Haemost (2004) 92:503-8. doi:10.1160/ TH04-04-0250

2. Renné T, Pozgajová $M$, Grüner S, Schuh K, Pauer $H$, Burfiend $P$, et al. Defective thrombus formation in mice lacking coagulation factor XII. J Exp Med (2005) 202:271-81. doi:10.1084/jem.20050664

3. Renné T, Schmaier AH, Nickel KF, Blomback M, Maas C. In vivo roles of factor XII. Blood (2012) 120:4296-303. doi:10.1182/blood-2012-07-292094

4. Gould TJ, Vu TT, Swystun LL, Dwivedi DJ, Mai SHC, Weitz JI, et al. Neutrophil extracellular traps promote thrombin generation through plateletdependent and platelet-independent mechanisms. Arterioscler Thromb Vasc Biol (2014) 34:1977-84. doi:10.1161/ATVBAHA.114.304114

5. Hess R, Wujak L, Hesse C, Sewald K, Jonigk D, Warnecke G, et al. Coagulation factor XII-regulated inflammatory responses in human lungs. Thomb Haemost (2017) 117:1896-907. doi:10.1160/TH16-12-0904

6. Bender L, Weidmann H, Rose-John S, Renné T, Long AT. Factor XII-driven inflammatory reactions with implications for anaphylaxis. Front Immunol (2017) 8:1115. doi:10.3389/fimmu.2017.01115

7. Gailani D, Bane CE, Gruber A. Factor XI and contact activation as targets for antithrombotic therapy. J Thromb Haemost (2015) 13:1383-95. doi:10.1111/jth.13005

8. Danese E, Montagnana M, Lippi G. Factor XII in haemostasis and thrombosis: active player or (innocent) bystander? Semin Thromb Hemost (2016) 42:682-8. doi:10.1055/s-0036-1571338

9. Wilmot H, Hockley J, Rigsby P, Gray E. Establishment of the World Health Organization $2^{\text {nd }}$ International Standard for Factor XI, Plasma, Human. Front Med (2017) 4:28. doi:10.3389/fmed.2017.00028

\section{ACKNOWLEDGMENTS}

The authors would like to thank the "Factor XI and the contact system" subcommittee of the SSC of the ISTH and all the participants of the study (Renata Zadro, University Hospital Center Zagreb, Croatia; Johannes Sidelmann, University of Southern Denmark, Esbjerg, Denmark; Caroline Lawrence and Grainne Hickman, Glasgow Royal Infirmary, Glasgow, United Kingdom; Sophie Desseauve and Jean Amiral, Hyphen Biomed, Neuville Sur Oise, France; Chris Gardiner and Ian Mackie, University College London, London, United Kingdom; Regina Gebauer and Michael Timme, Siemens Healthcare Diagnostics Products GmbH, Marburg, Germany; Anne Riddell, Royal Free London NHS Hospital Trust, London, United Kingdom; Frédéric Dhainaut, LFB, Cedex, France; Aurelie Hazotte, LFB, Cedex, France; Roger Luddington, Addenbrooke's Hospital, Cambridge, United Kingdom; Steffen Kistner, Jens Daufenbach and Karin Fuchs, Biotest AG, Dreieich, Germany; Mariona Bono and Begona Alonso, Diagnostic Grifols SA, Barcelona, Spain; Katherine Tull, Peter Vandeberg, Maria Cruz, Catherine Russ, Grifols Inc., NC, USA; Denise Foulon, Affinity Biologicals, ON, Canada; Colin Downey, Royal Liverpool Hospital, Liverpool, United Kingdom; Annette Feussner, CSL Behring GmbH, Marburg, Germany; Carel Eckmann and Jeanette Rentenaar, Sanquin Blood Supply, Amsterdam, Netherlands; Kathleen Trumbull, Instrumentation Laboratory, MA, USA; Annette Bowyer, Royal Hallamshire Hospital, Sheffield, United Kingdom; Nikolaus Binder, Technoclone GmbH, Vienna, Austria; Stella Williams, NIBSC, United Kingdom; Mikhail Ovanesov, Yideng Liang, Tseday Tegegn, and Ravi Rasmi Jasti, FDA/CBER, MD, USA; Claire Martin, Anne Lochu, Jerome Beltran, Marc Grimaux, and Francois Nicham, Stago, Gennevilliers, France).

10. Hubbard A, Bevan S, Matejtschuk P. Impact of residual moisture ad formulation on factor VIII and factor V recovery in lyophilised plasma reference materials. Anal Bioanal Chem (2007) 387:2503-7. doi:10.1007/ s00216-006-0855-x

11. Campbell PJ. Procedures used for the production of biological standards and reference preparations. J Biol Stand (1974) 2:259-67. doi:10.1016/00921157(74)90034-1

12. Activated coagulation factors. Eur Pharm (2016) monograph 01/2008:20622. Available fron: http://online.pheur.org/EN/entry.htm

13. Prekallikrein activator. Eur Pharm (2017) monograph 04/2016:20615. Available from: http://online.pheur.org/EN/entry.htm

14. Madsen DE, Sidelmann JJ, Overgaard K, Koch C, Gram JB. ELISA for determination of total coagulation factor XII concentration in human plasma. J Immunol Methods (2013) 394:32-9. doi:10.1016/j.jim.2013.04.012

15. Finney DJ. Statistical Methods in Biological Assay. 3rd ed. London: Charles Griffin (1978).

16. Grubbs F. Procedures for detecting outlying observations in samples. Technometrics (1969) 11:1-21. doi:10.1080/00401706.1969.10490657

Conflict of Interest Statement: The authors declare that the research was conducted in the absence of any commercial or financial relationships that could be construed as a potential conflict of interest.

Copyright (c) 2018 Wilmot, Hockley, Rigsby and Gray. This is an open-access article distributed under the terms of the Creative Commons Attribution License (CC BY). The use, distribution or reproduction in other forums is permitted, provided the original author(s) and the copyright owner are credited and that the original publication in this journal is cited, in accordance with accepted academic practice. No use, distribution or reproduction is permitted which does not comply with these terms. 